MYU Tokyo

S \& M 1382

\title{
Low-Noise Chopper-Stabilized Resistive Readout Integrated Circuit with Ripple Rejection Loop
}

\author{
Youngwoon Ko, Hyungseup Kim, Yunjong Park, \\ Yeongjin Mun, Dong-il "Dan" Cho, ${ }^{1}$ and Hyoungho Ko \\ Department of Electronics Engineering, Chungnam National University, Daejeon 34134, Republic of Korea \\ ${ }^{1}$ Department of Electrical and Computer Engineering, Seoul National University, Seoul 08826, Republic of Korea
}

(Received March 31, 2016; accepted February 27, 2017)

Keywords: chopper stabilization, resistive readout circuit, ripple rejection loop, resistive sensor

Resistive sensors are widely used in many applications such as pressure sensors, touch screens, body composition analyzers, and acceleration sensors. Chopper stabilization is an effective solution to reduce DC offset and low-frequency flicker noises. In the demodulation stage of chopper stabilization, a high-order low-pass filter, which occupies a large area, is generally required to attenuate the up-converted DC offset and low frequency noises or "ripples". We propose a lownoise chopper-stabilized resistive readout integrated circuit (IC) with a ripple rejection loop (RRL). When the RRL operates, the modulated ripple is demodulated to the baseband. The demodulated ripple is integrated using a Miller integrator. The output of the integrator is chopped and fedback negatively to the input of the instrumentation amplifier (IA). The Miller integrator is implemented using MOS-bipolar pseudoresistors and feedback capacitors to achieve high CMRR, high DC rejection, and a small circuit size. The chip is fabricated using a $0.18-\mu \mathrm{m}$ complementary metaloxide-semiconductor (CMOS) process with an active area of $1.8 \mathrm{~mm}^{2}$. The power consumption is $827 \mu \mathrm{W}$ at $3.3 \mathrm{~V}$ supply. The input referred noise is measured at $0.37 \mu \mathrm{Vrms}$ in the frequency band of $1-200 \mathrm{~Hz}$.

\section{Introduction}

With the development of smart devices for the Internet of Things (IoT), resistive sensors based on Wheatstone bridge configurations and resistive readout integrated circuits (ICs) are extensively used in various application areas such as pressure sensors, touch screens, body impedance analyzers, and acceleration sensors. ${ }^{(1-3)}$ In general, the resistive readout IC amplifies the voltage generated from the Wheatstone bridge using a precise instrumentation amplifier (IA). In previous papers, chopper current feedback IAs and differential difference IAs applying a chopper stabilization technique have been used to achieve high CMRR and low DC offset. ${ }^{(4,5)}$ To obtain low noise readout characteristics, chopper stabilization and the auto-zeroing techniques are commonly used. These methods reduce DC offset and low-frequency flicker noise. Because the auto-zero architecture poses problems of switching noises as well as noise folding due to samplinginduced high frequency aliasing, the chopper stabilized architecture is preferred in low noise and low power continuous-time applications. ${ }^{(4,5)}$

"Corresponding author: e-mail: hhko@cnu.ac.kr

http://dx.doi.org/10.18494/SAM.2017.1566

ISSN 0914-4935 C MYU K.K. 
In the modulation stage of the chopper stabilization technique, input signals modulate the carrier frequency, and they are processed at a high frequency with less noise. However, the offset voltage of an IA also modulates the carrier signal, which appears as a signal called a "ripple," at the output of the demodulation stage of chopper stabilization. A sample-and-hold filter ${ }^{(6,7)}$ and a highorder low-pass filter (LPF) can be used to attenuate ripple voltage. The sample-and-hold filter, however, still presents a noise folding problem. A high-order LPF generally takes up too large an area.

In this paper, we present a low-noise resistive readout IC using a chopper stabilized IA with an improved ripple rejection loop (RRL). ${ }^{(2,8)}$ The proposed IC uses the chopper stabilization technique to reduce DC offset and low-frequency flicker noise. The RRL effectively reduces the ripple voltage and DC offset while taking up only a small area. The circuit design and the experimental evaluation of the resistive readout IC are discussed.

\section{Proposed Chopper-Stabilized Resistive Readout IC with RRL}

\subsection{Proposed architecture and operating principle of wind flow sensor}

The architecture of the proposed readout IC for processing signals from the piezoresistive micro-electromechanical systems (MEMS) sensor is illustrated in Fig. 1. The readout IC includes an IA, an RRL, an LPF, and a successive approximation register (SAR) analog-to-digital converter (ADC).

To validate the performance of the IC, a wind flow sensor on a piezoresistive Wheatstone bridge is used as shown in Fig. 2. All resistive sensor elements of this bridge are designed to change by $\Delta R$ during wind flow to eliminate the linearity error. ${ }^{(9)}$ The output voltage is expressed as

$$
\begin{aligned}
& V_{o}=\left(\frac{R+\Delta R}{R-\Delta R+R+\Delta R}-\frac{R-\Delta R}{R+\Delta R+R-\Delta R}\right) V_{D D} \\
& V_{o}=\frac{\Delta R}{R} V_{D D} .
\end{aligned}
$$

An IA implemented with three op-amps is used to amplify the modulated small differential input voltage from the wind flow sensor. The IA has two non-inverting amplifiers in the first stage

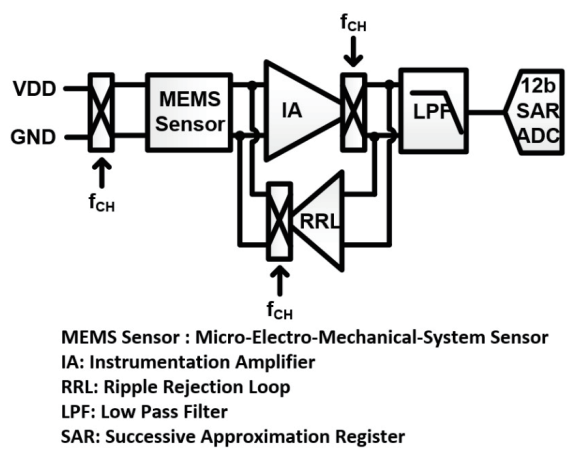

Fig. 1. Architecture of the proposed resistive readout IC.

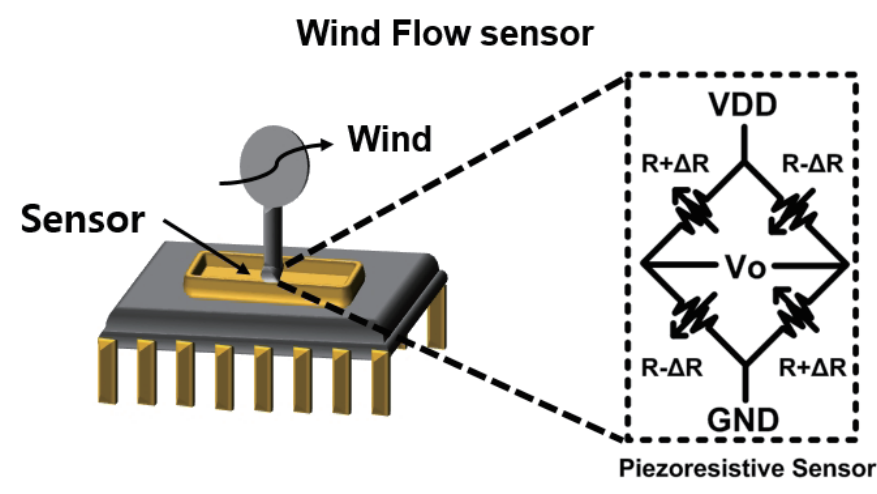

Fig. 2. (Color online) Wind flow sensor on piezoresistive Wheatstone bridge. 
and a differential inverting amplifier in the second stage; it presents high input impedance and a large gain. The offset and 1/f noise components in the low-frequency band are moved to the highfrequency band by chopper modulation. To reduce the ripples created by the offset in the output voltage, an RRL is used in this IC. The RRL operates as a notch filter at the chopper frequency. Thus, the requirements for higher-order LPF are relaxed. Glitches caused by the chopping and high frequency noise that remain in the IA output are removed by a fourth-order Sallen-Key LPF. The output differential signal of the LPF is converted into digital code by a 12-bit SAR ADC.

\subsection{Chopper-stabilized resistive readout IC with RRL}

Figure 3 shows the proposed chopper stabilized resistive readout IC with RRL. The operating principle of the IC is as follows. The top and bottom nodes of the resistive wind flow sensor are driven by a modulating chopper (CH1). The $\mathrm{CH} 1$ is controlled by periodic switches $\mathrm{P} 1$ and $\mathrm{P} 2$. The three op-amps in the IA are used to amplify the modulated, small differential input voltage from the wind flow sensor. The IA transfer function is expressed as

$$
\frac{\text { Vout }}{I N P-I N N}=\left(1+\frac{2 R_{f_{1}}}{R_{c}}\right)\left(\frac{R_{f_{2}}}{R_{\text {in }}}\right) \text {. }
$$

The gain of this IA can be controlled from $40-100 \mathrm{~dB}$ by adjusting $R_{c}$ in the first stage and $R_{\text {in }}$ in the second stage. In addition, the offset is corrected by the current steering digital-to-analog converter (DAC) with binary weighted control. The amplified differential signals are demodulated by a chopper (CH2). At the same time, the input referred offset of the IA is also modulated by the chopper (CH2). This chopping of the offset voltage can create large ripples. To reduce the ripples in the output voltage, the RRL is proposed as shown in Fig. 4. The proposed RRL is composed of a fully differential Miller integrator, which uses MOS-bipolar pseudoresistors and capacitors in the negative feedback loop. ${ }^{(2,8,10)}$ The transfer function of the Miller integrator is expressed as

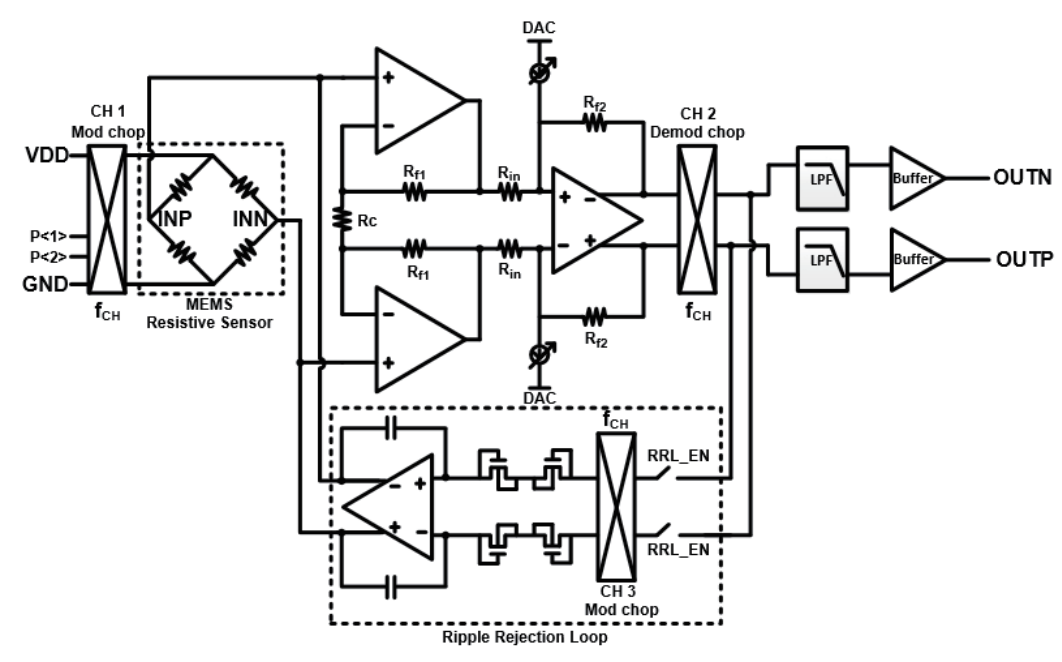

Fig. 3. Chopper-stabilized resistive readout IC with RRL. 


$$
\frac{O U T P-O U T N}{I N P-I N N}=-\frac{1}{S} \times \frac{C_{f}}{R_{\text {in }}} .
$$

The modulated offset ripple is demodulated to the baseband. The demodulated ripple is integrated as governed by Eq. (3). The output of the Miller integrator is demodulated to a low frequency by a chopper (CH3) and transferred into the input stage of the IA through a negative feedback loop. As a result, this RRL effectively reduces the ripple and compensates for the DC offset.

\section{Experimental Results}

The proposed chopper-stabilized resistive readout IC with RRL was fabricated using a $0.18-\mu \mathrm{m}$ 1P6M complementary metal-oxide-semiconductor (CMOS) process with an active area of $1.8 \mathrm{~mm}^{2}$ excluding the I/O pads. A photograph of the fabricated die form of the IC with RRL is shown in Fig. 5. Figure 6 shows the setup used for evaluation of the chopper-stabilized resistive readout IC along

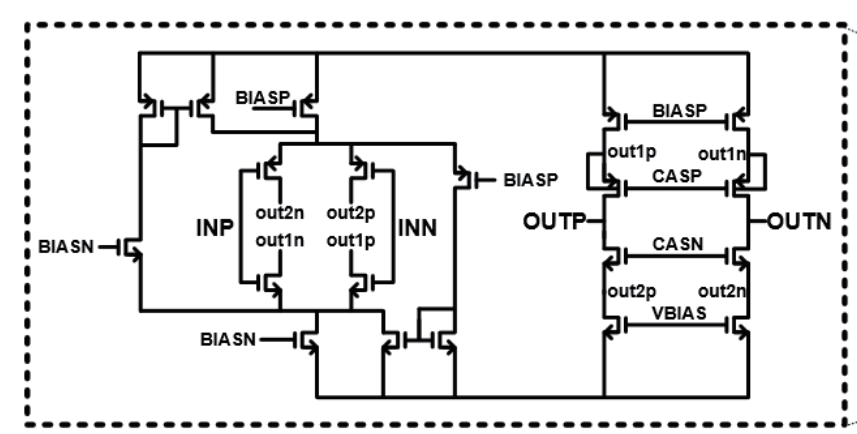

Fully differential op-amp for Miller Integrator

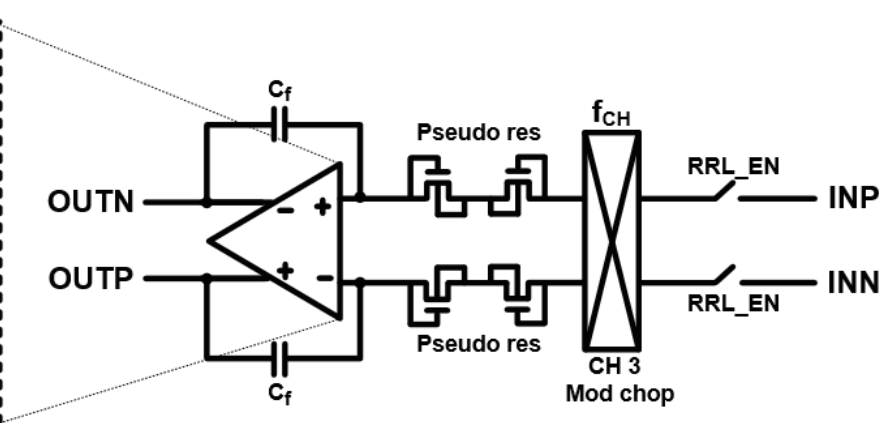

Ripple Rejection Loop

Fig. 4. RRL circuit.

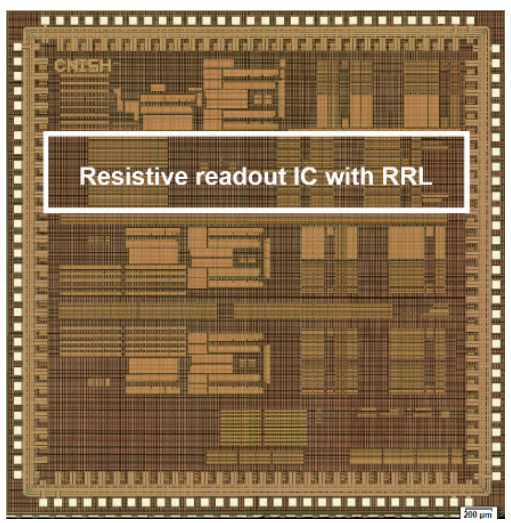

Fig. 5. (Color online) Die photograph of chopper-stabilized resistive readout IC with RRL.

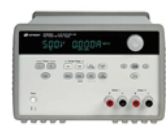
DC power supply
Keysight E3648A 3.3-V power supply

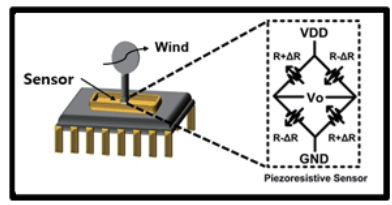

Wind flow sensor
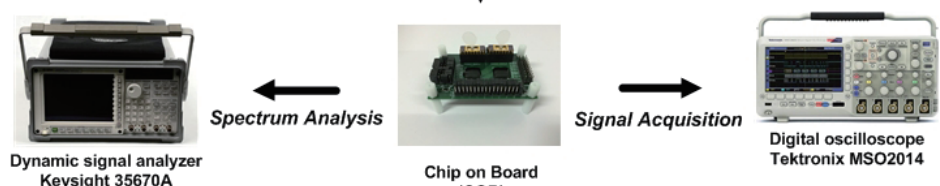

Keysight $35670 \mathrm{~A}$
Kerc signal analyz
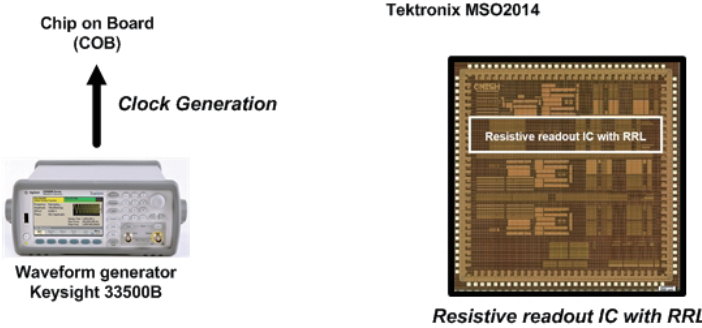

Fig. 6. (Color online) Evaluation setup for the chopper-stabilized resistive readout IC. 
with the two resistive wind flow sensors. The two wind flow sensors are mounted perpendicularly to each other on the PCB.

Figure 7 shows the transfer function of the chopper-stabilized resistive readout IC. At the default gain setting of the IC, the gain measured by the spectrum analyzer is $42.2 \mathrm{~dB}$. Furthermore, the cut-off frequency of the transfer function is approximately $200 \mathrm{~Hz}$. The results of the measurement of input referred resistive noise are shown in Fig. 8. After measuring the output referred noise with a spectrum analyzer, input referred noise is determined by dividing the output referred noise by the gain of the resistive IC. When the chopper is turned off, the input referred noise is $1.5 \mu \mathrm{Vrms}$, as shown in Fig. 8(a). When the chopper is turned on at a frequency of $32 \mathrm{kHz}$, the input referred noise is $0.37 \mu \mathrm{Vrms}$ for a bandwidth of $200 \mathrm{~Hz}$, as shown in Fig. 8(b).

Figure 9 shows the output of the resistive readout IC with RRL. On the left side of the figure, the DC offset can be seen on the waveforms when the RRL is disabled. However, when the RRL is enabled, it can be seen that the offset and the ripple that occurred due to chopper operation are automatically calibrated within a small calibration time of $1.2 \mathrm{~s}$.

The time domain outputs of the chopper-stabilized resistive readout IC as measured when connected to wind flow sensors are shown in Fig. 10. The external air flow at $3 \mathrm{~Hz}, 5 \mathrm{~cm} / \mathrm{s}$ is applied to the wind flow sensor board. The output signal of the first wind flow sensor, which is placed in sensitive directions, varies with the external air flow. The output signal of the second wind flow sensor, which is placed perpendicularly to the first sensor, is almost fixed at the value of the DC bias. The total power consumption of the IC is $827 \mu \mathrm{W}$ with a $3.3 \mathrm{~V}$ supply. The performance comparisons of the readout IC with previously reported values are summarized in Table 1.

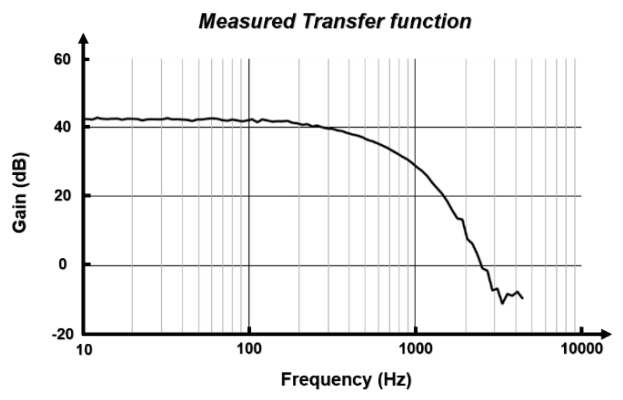

Fig. 7. Transfer function of the chopper-stabilized resistive readout IC.

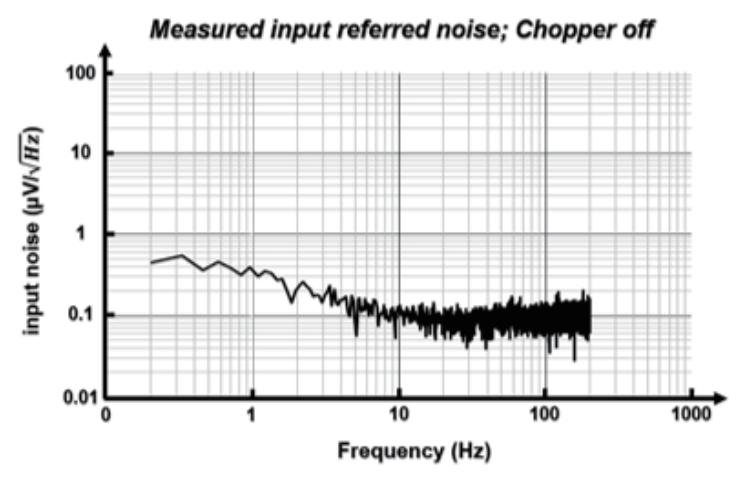

(a)

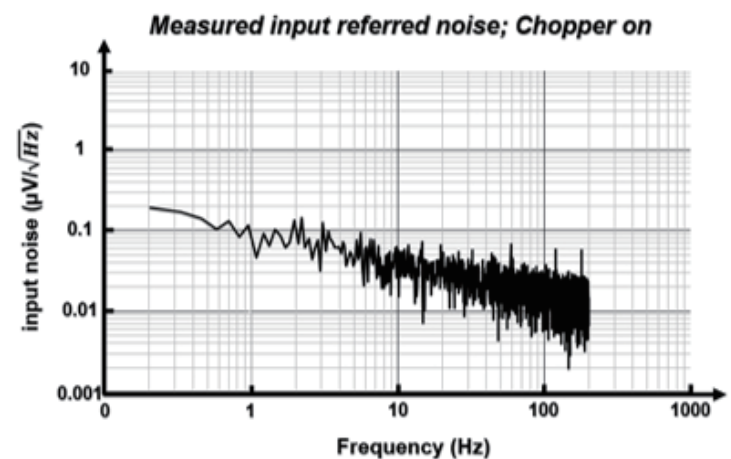

(b)

Fig. 8. Input referred noise of the chopper-stabilized resistive readout IC. 


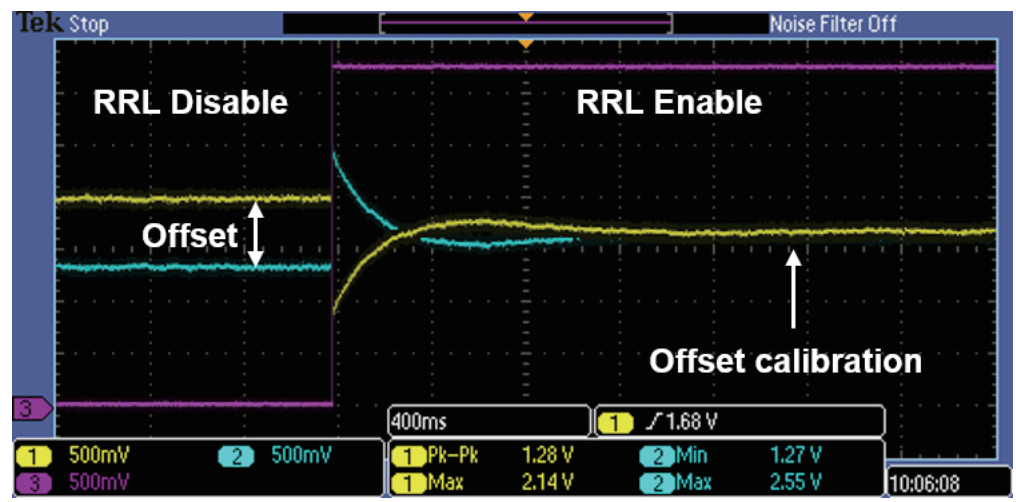

Fig. 9. (Color online) Output of resistive readout IC with RRL.

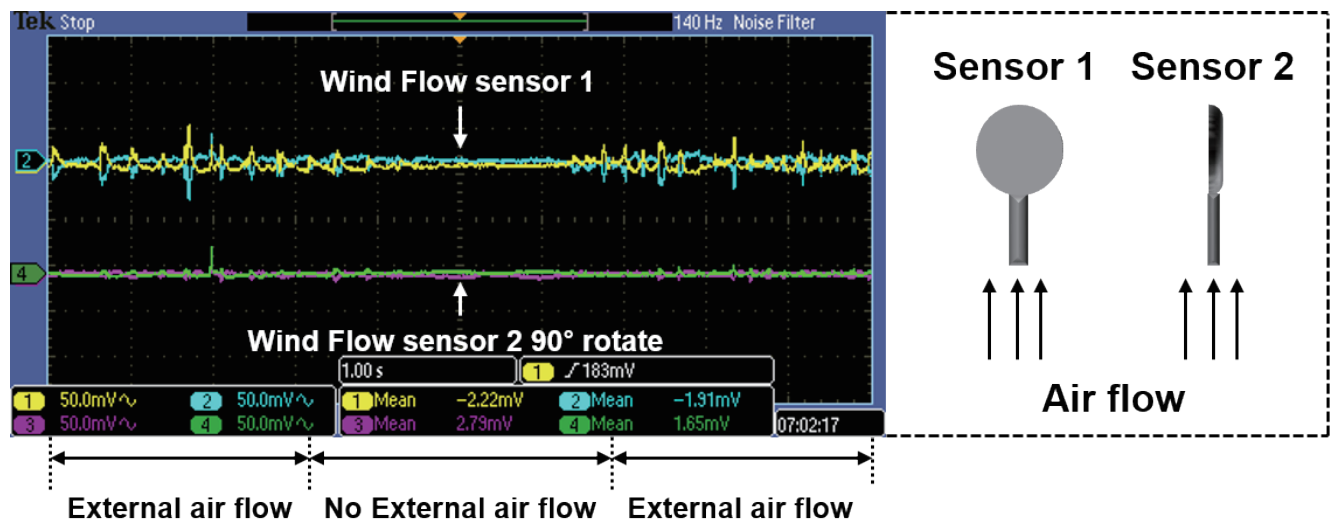

Fig. 10. (Color online) Time domain outputs of the chopper-stabilized resistive readout IC.

Table 1

Performance summary of measured parameters.

\begin{tabular}{lcccc}
\hline Parameter & This work & Ref. 4 & Ref. 11 & Ref. 12 \\
\hline Technology $(\mu \mathrm{m})$ & 0.18 & 0.18 & 0.18 & 0.065 \\
Supply $(\mathrm{V})$ & 3.3 & 2.7 & 1.8 & 1 \\
Power $(\mu \mathrm{W})$ & 827 & 270 & 62 & 12.3 \\
Bandwidth $(\mathrm{kHz})$ & 0.2 & 2 & $4.5-9$ & 0.040 \\
Input Referred Noise $(\mu \mathrm{Vrms})$ & 0.37 & 3.76 & 1.8 & 11 \\
Active area $\left(\mathrm{mm}^{2}\right)$ & 1.8 & - & 0.3 & 0.358 \\
Low noise technique & Chopper & Chopper & Chopper & Correlated \\
RRL & stabilization & stabilization & stabilization & double sampling \\
\hline & $\mathrm{Y}$ & $\mathrm{N}$ & $\mathrm{N}$ & $\mathrm{N}$ \\
\hline
\end{tabular}

\section{Conclusions}

A low-noise chopper-stabilized resistive readout IC with RRL for use with resistive sensors is presented. The chip is fabricated using a $0.18-\mu \mathrm{m}$ 1P6M CMOS process with an active area of 1.8 $\mathrm{mm}^{2}$. This resistive readout IC consumes $827 \mu \mathrm{W}$ with a $3.3 \mathrm{~V}$ supply. The input referred noise is 0.37 $\mu \mathrm{Vrms}$ for a bandwidth of $200 \mathrm{~Hz}$. Measurements demonstrated that the resistive readout IC with RRL can achieve low noise and can reduce ripples. 


\section{Acknowledgments}

This research was supported by a grant to the Bio-Mimetic Robot Research Center (UD130070ID) funded by the Defense Acquisition Program Administration.

\section{References}

1 H. Ko, H. Song, S. Im, H. Kim, B. Jang, H. Shim, and D. Cho: Sens. Mater. 27 (2015) 437.

2 Y. Park, H. Song, H. Kim, and H. Ko: Proc. Int. SoC Design Conference (IEEE, Gyungju, 2015) 229.

3 H. Ha, Y. Suh, S. K. Lee, H. Park, and J. Y. Sim: Proc. CICC (IEEE, San Jose, 2012) 1.

4 G. T. Ong and P. K. Chan: IEEE Trans. Instrum. Meas. 63 (2014) 2253.

5 R. Wu, K. A. A. Makinwa, and J. H. Huijsing: IEEE J. Solid-State Circuits 44 (2009) 3232.

6 R. Burt and J.Zhang: IEEE J. Solid-State Circuits 41 (2006) 2729.

7 A. Bakker and J. H. Huijsing: Proc. 23rd European Solid-State Circuits Conference (IEEE, 1997) 200.

8 J. Wu, M. K. Law, P. I. Mak, and R. P. Martins: IEEE Trans. Circuits. Syst. II 63 (2016) 351.

9 G. Graaf and R. F Wolffenbuttel: Proc. 30th European Solid-State Circuits Conference (IEEE, Leuven, 2004) 451.

10 R. R. Harrison and C. Charles: IEEE J. Solid-State Circuits 38 (2003) 958.

11 A. Pun, J. Wong, G. Chan, W. Wong, D. Kwong, and K. Wang: Proc. Int. Symp. Circuits and Systems (IEEE, 2013) 1500 .

12 K. C. Koay and P. K. Chan: IEEE Trans. Circuits. Syst. I (2016) 1. 